\title{
Association of Endocrine Therapy and Dementia in Women with Breast Cancer
}

This article was published in the following Dove Press journal:

Breast Cancer: Targets and Therapy

\author{
Mikayla R Thompson' \\ Jiangong $\mathrm{Niu}^{2}$ \\ Xiudong Lei ${ }^{2}$ \\ Malgorzata Nowakowska ${ }^{3}$ \\ Mackenzie R Wehner ${ }^{2,4}$ \\ Sharon H Giordano ${ }^{2,5}$ \\ Kevin T Nead (1D ${ }^{6,7}$ \\ 'Department of Epidemiology and \\ Environmental Health, University at \\ Buffalo School of Public Health and \\ Health Professions, Buffalo, NY, USA \\ ${ }^{2}$ Department of Health Services \\ Research, University of Texas MD \\ Anderson Cancer Center, Houston, TX, \\ USA; ${ }^{3}$ Department of Medicine, Baylor \\ College of Medicine, Houston, TX, USA; \\ ${ }^{4}$ Department of Dermatology, University \\ of Texas MD Anderson Cancer Center, \\ Houston, TX, USA; ${ }^{5}$ Department of \\ Breast Medical Oncology, University of \\ Texas MD Anderson Cancer Center, \\ Houston, TX, USA; ${ }^{6}$ Department of \\ Epidemiology, University of Texas MD \\ Anderson Cancer Center, Houston, TX, \\ USA; ${ }^{7}$ Department of Radiation \\ Oncology, University of Texas MD \\ Anderson Cancer Center, Houston, \\ TX, USA
}

Correspondence: Kevin T Nead

Tel + I 713 563-5155

Email ktnead@mdanderson.org
Purpose: Prior studies have reported differing results regarding the association between endocrine therapy (ET) in the treatment of breast cancer and dementia risk. However, existing findings may be limited by common sources of bias and confounding. Here we investigate the association of ET utilized in the definitive setting to treat non-metastatic breast cancer with dementia risk accounting for multiple potential sources of bias and confounding.

Patients and Methods: We conducted a retrospective study in SEER-Medicare of women aged $\geq 66$ years with non-metastatic breast cancer. We examined the risk of all-cause dementia among ET users versus non-ET users using multivariable regression models, accounting for the competing risk of death, and using a start of the follow-up period as 12-months following breast cancer diagnosis for both groups to avoid immortal time bias.

Results: Among 25,777 individuals there were 2,869 incident dementia cases. We found a statistically significantly decreased risk of any dementia among ET users in unadjusted and adjusted models that completely attenuated when accounting for the competing risk of death (hazard ratio, 0.98; 95\% confidence interval, 0.90-1.07).

Conclusion: When accounting for common sources of bias and confounding we did not find evidence to support an association between ET in the definitive treatment of non-metastatic breast cancer and dementia risk. These results suggest that ET may not be associated with dementia risk.

Keywords: breast cancer, endocrine therapy, dementia, aromatase inhibitors, selective estrogen receptor modulators

\section{Introduction}

Globally, breast cancer is the most common type of cancer diagnosed in women with over 270,000 new cases estimated in 2020 in the United States alone. ${ }^{1}$ However, despite this high incidence, the mortality rate for breast cancer remains low. Therefore, side effects from common treatments for breast cancer can have significant long-term public health implications.

Endocrine therapy (ET), which works to decrease the interaction of circulating hormones estrogen and progesterone with hormone-receptor positive breast cancer cells, is a mainstay of treatment for breast cancer that has been shown to reduce breast cancer mortality rates. ${ }^{2}$ Broadly, endocrine therapy typically includes selective estrogen receptor modulators (SERMs) and aromatase inhibitors (AIs). SERMS work by blocking the effect of estrogens in tissues and AIs work by preventing the enzymatic conversion of other hormones in the body into estrogen. Multiple epidemiological studies have shown an association between estrogens and cognitive health, including 
altered dementia risk associated with ET use to treat breast cancer. A nationwide population-based study in Taiwan among patients diagnosed with breast cancer found that tamoxifen users had a $17 \%$ relative reduction in dementia risk. $^{3}$ Conversely, a Danish nationwide medical registries study among patients with breast cancer found no clear association between tamoxifen use and dementia risk. ${ }^{4}$ A recent study using Humana claims data examined 326,485 women with breast cancer and found a $12 \%$ relative dementia risk reduction. ${ }^{5}$ However, this and prior studies are limited by potential sources of bias through the inclusion of heterogenous populations (composition bias) such as those with local and metastatic disease, incompletely accounting for immortal time (immortal time bias), and the competing risk of death (competing risk bias). ${ }^{6-8}$ Here we utilize data from SEER-Medicare to investigate the association of ET utilized in the definitive setting to treat non-metastatic breast cancer with dementia risk accounting for multiple potential sources of bias and confounding.

\section{Methods}

We conducted a retrospective study in the Surveillance, Epidemiology and End Results (SEER)-Medicare linked dataset of women aged 66 year of age or older with nonmetastatic breast cancer diagnosed from 2008-2015 (Figure 1). SEER-Medicare is a linkage dataset of cancer registries data containing clinical, demographic and cause of death information with Medicare claims data for covered health care services.

We compared ET users (received ET in the 12 months following breast cancer diagnosis per Part D claims data) to non-ET users. We defined ET as 1) AI only (anastrozole, exemestane, letrozole), 2) SERM only (tamoxifen, toremifene), and 3) AI and SERM. The primary outcome was incident all-cause dementia. Secondary analyses examined ET type (selective estrogen receptor modulators [SERMs], aromatase inhibitors) and dementia type (vascular dementia, Alzheimer's dementia, other or unclassified dementia). The start of the follow-up period was 12-months following breast cancer diagnosis for both groups to avoid immortal time bias. ${ }^{6}$ Baseline patient characteristics were compared using a $t$-test or $\chi^{2}$ test. Hazard ratios (HRs) were calculated using multivariable adjusted Cox proportional hazards regression models with death from any cause as a competing event using the method of Fine and Gray. ${ }^{9}$ Models were adjusted for age, race, region, marital status, $\mathrm{T}$ stage, $\mathrm{N}$ stage, Charlson Comorbidity Index, treatment with radiation, surgery, or chemotherapy. We conducted sensitivity analyses using one-to-one nearest-neighbor propensity score matching. This study was institutional review board approved by MD Anderson Cancer Center and followed the Strengthening the Reporting of Observational Studies in Epidemiology (STROBE) reporting guideline. Tests were considered to be significant with a 2 -sided $\mathrm{P}<.05$. We used SAS version 9.4 and $\mathrm{R}$ version 4.0.0 to perform the analyses.

\section{Results}

The analytic cohort (Figure 1) included 25,777 individuals with median follow-up of 32 months (interquartile range, 32-78 months). Compared to the no-ET group $(n=6,649)$, the ET group $(n=19,128)$ was younger $(74$ versus 76 years), more likely to be white ( $88 \%$ versus $87 \%$ ), married ( $47 \%$ versus $40 \%)$, have T1 disease $(68 \%$ versus $66 \%)$, be node positive ( $24 \%$ versus $18 \%)$, undergo radiation therapy ( $65 \%$ versus $53 \%)$, and not undergo chemotherapy ( $22 \%$ versus $34 \%$ ), $\mathrm{p}<0.001$ for all (Table 1). There were 2,869 incident dementia cases including vascular dementia $(\mathrm{n}=159)$, Alzheimer's $(n=475)$ and other or unclassified dementia $(n=2,235)$. $10.2 \%$ of individuals in the ET group and $13.9 \%$ of individuals in the no-ET were classified as having dementia in our study. We found a statistically significantly decreased risk of any dementia among ET users in unadjusted models (Table 2) that attenuated when accounting for confounders and the competing risk of death (HR, 0.98; 95\% confidence interval [CI], 0.90-1.07). Results were consistent when examining vascular dementia (HR, 0.93; 95\% CI, 0.74-1.16), Alzheimer's (HR, 0.99; 95\% CI $0.87-1.15$ ), and other or unclassified dementia (HR 0.98; 95\% CI 0.90-1.08). Association of ET subtype according to ET class are presented in Supplemental Table 1. Sensitivity analysis using propensity score matching to examine ET use with any dementia showed similar results (HR 0.95 ; 95\% CI 0.86-1.03). When stratifying our analysis by type of ET we observed an increased risk of dementia with SERMs (Table 2) that was no longer statistically significant with propensity score matching (HR, 1.16; 95\% CI 0.99-1.35; $\mathrm{p}=0.056$ ).

\section{Discussion}

We did not find evidence to support an association between ET in the definitive treatment of non-metastatic breast cancer and dementia risk, which differs from some prior studies showing a protective effect. ${ }^{3,5}$ We found that 


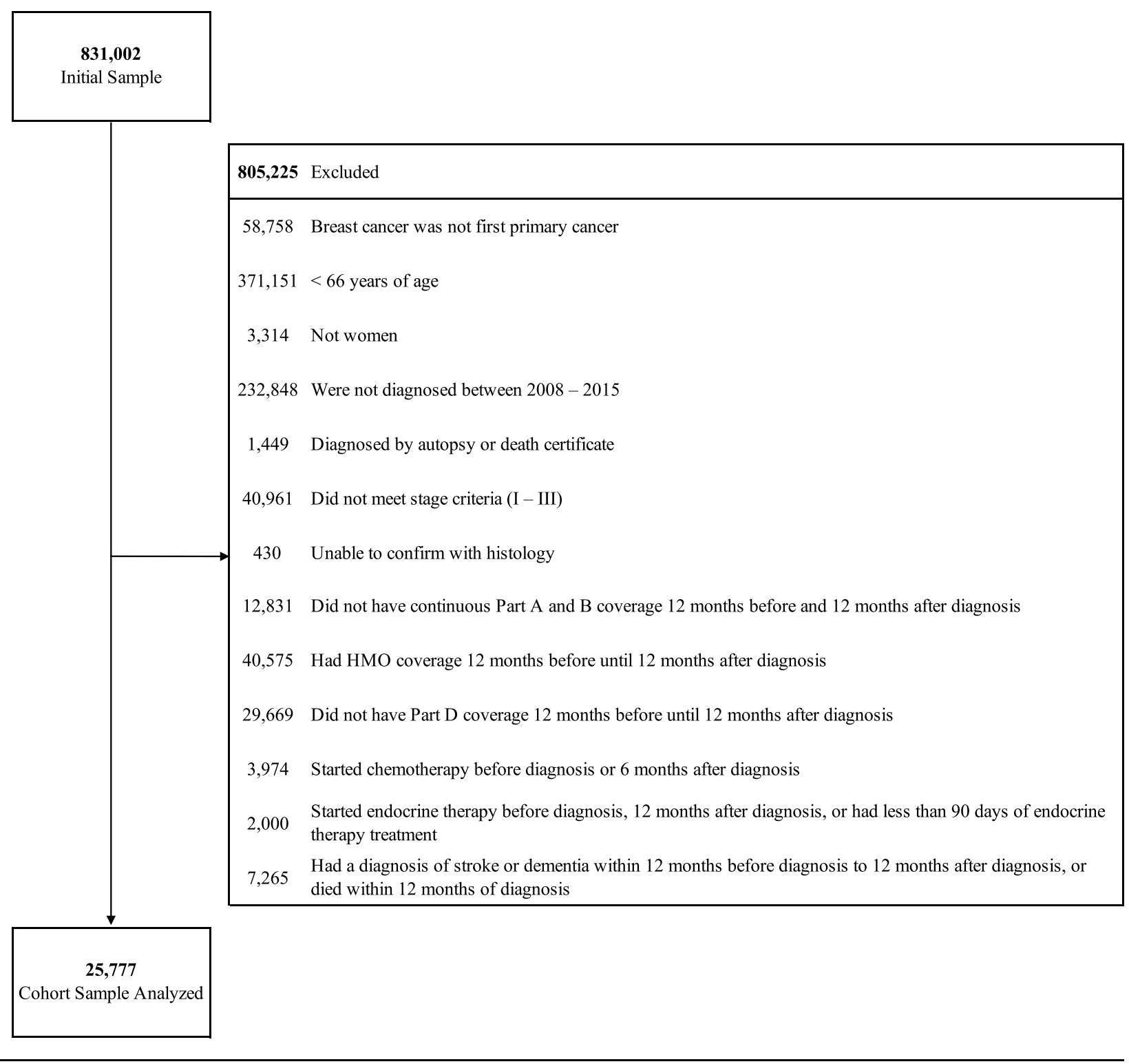

HMO, health maintenance organization; SEER,Surveillance, Epidemiology and End Results

Figure I Flowchart with exclusion criteria to identify analytic cohort.

$10.2 \%$ of individuals in the ET group and $13.9 \%$ of individuals in the no-ET had dementia, which is in line with the $11.5 \%$ of Medicare Fee-for-Service beneficiaries aged $\geq 65$ years with dementia. ${ }^{10}$ Our analysis showed a protective effect of ET on dementia in fully adjusted models that completely attenuated when accounting for the competing risk of death, which is critical when examining incident outcomes in older individuals ${ }^{7}$ and was not accounted for in all prior studies. Furthermore, our use of a relatively homogenous cohort may be less susceptible to selection bias, ${ }^{8}$ and we designed our analysis to limit the impact of immortal time bias. ${ }^{6}$
Our study accounted for the competing risk of death when analyzing the association of ET use and dementia risk and this may primarily explain the difference between our results and those observed in prior studies showing a protective effect. ${ }^{5}$ A competing risk is an alternative outcome, such as death, that alters the probability of the outcome of interest. In traditional survival analyses individuals who are censored for any reason are considered at risk for the primary outcome for the remaining duration of the study. However, while individuals who are censored for being lost to follow-up may remain at risk for dementia, individuals who become deceased are no longer at risk 
Table I Demographics

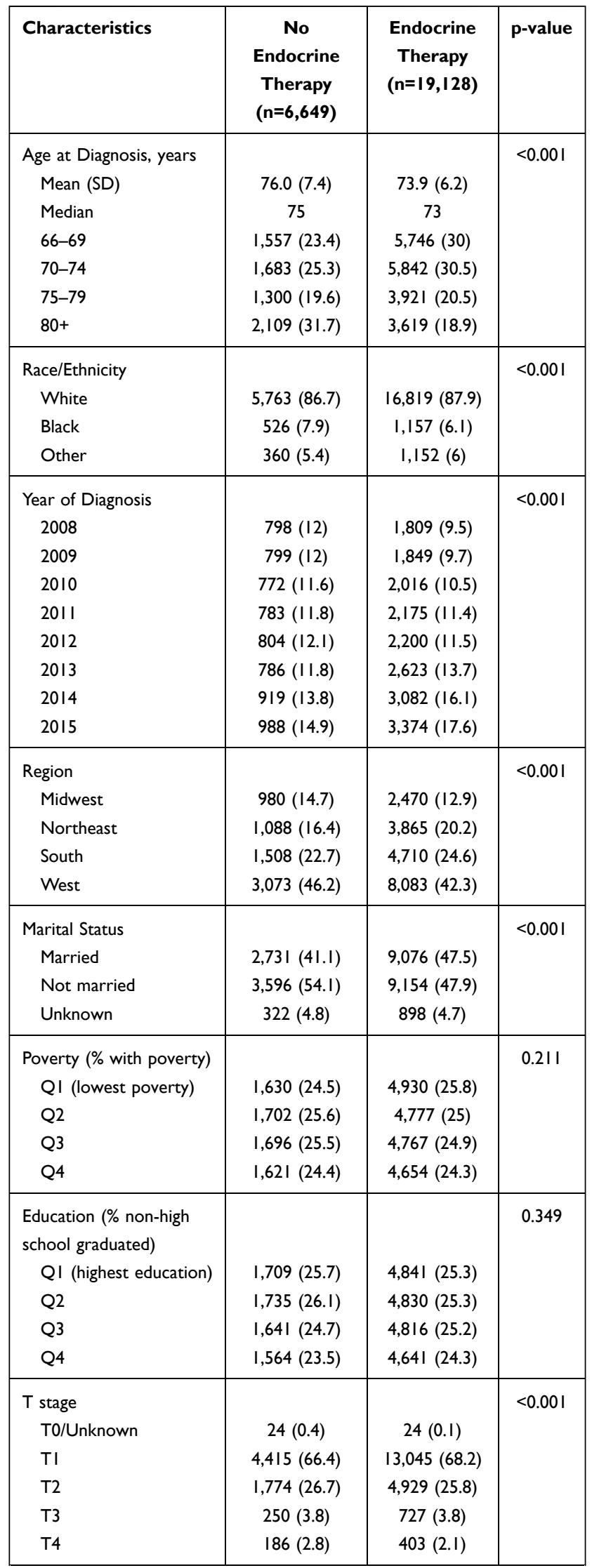

(Continued)
Table I (Continued).

\begin{tabular}{|c|c|c|c|}
\hline Characteristics & $\begin{array}{c}\text { No } \\
\text { Endocrine } \\
\text { Therapy } \\
(n=6,649)\end{array}$ & $\begin{array}{c}\text { Endocrine } \\
\text { Therapy } \\
(n=19,128)\end{array}$ & p-value \\
\hline $\mathrm{N}$ stage & & & $<0.001$ \\
\hline No & $5,440(81.8)$ & 14,552 (76.I) & \\
\hline $\mathrm{NI}$ & $873(13.1)$ & $3,477(18.2)$ & \\
\hline N2 & $205(3.1)$ & $724(3.8)$ & \\
\hline N3 & $125(1.9)$ & $353(1.9)$ & \\
\hline Unknown & $6(0.1)$ & $22(0.1)$ & \\
\hline AJCC stage & & & 0.102 \\
\hline I & $4,119(62)$ & $|I, 58|(60.5)$ & \\
\hline ॥ & $2,005(30.2)$ & $5,931(31)$ & \\
\hline III & $525(7.9)$ & I,616 (8.5) & \\
\hline ER status & & & $<0.001$ \\
\hline Positive & $3,295(49.6)$ & 18,554 (97) & \\
\hline Negative & $3,139(47.2)$ & $245(1.3)$ & \\
\hline Unknown & $215(3.2)$ & $329(1.7)$ & \\
\hline Subtype & & & $<0.001$ \\
\hline $\mathrm{HR}+/ \mathrm{HER} 2-$ & $2,234(33.6)$ & $13,333(69.7)$ & \\
\hline HR+/HER2+ & $200(3)$ & $\mathrm{I}, 243(6.5)$ & \\
\hline HR-/HER2+ & $553(8.3)$ & $38(0.2)$ & \\
\hline HR-/HER2- & I,688 (25.4) & $72(0.4)$ & \\
\hline Unknown & $1,974(29.7)$ & $4,442(23.2)$ & \\
\hline Charlson Comorbidity & & & 0.720 \\
\hline Scores & & & \\
\hline 0 & $4,023(60.5)$ & $11,623(60.8)$ & \\
\hline 1 & I,575 (23.7) & $4,56 \mid(23.8)$ & \\
\hline $2+$ & I,05I (I5.8) & $2,944(15.4)$ & \\
\hline Type of Endocrine & & & $<0.001$ \\
\hline Therapy (ET) & & & \\
\hline None/Never & $6,649(100)$ & $0(0)$ & \\
\hline Selective Estrogen & $0(0)$ & I,762 (9.2) & \\
\hline Receptor Modulators & & & \\
\hline (SERM) only & & & \\
\hline Aromatase inhibitors & $0(0)$ & I5,058 (78.7) & \\
\hline $\begin{array}{l}\text { only } \\
\text { SERM + Al }\end{array}$ & $0(0)$ & $2,308(12.1)$ & \\
\hline Chemotherapy use & $2,287(34.4)$ & $4,250(22.2)$ & $<0.001$ \\
\hline Radiation Therapy use & $3,576(53.8)$ & I2,574 (65.7) & $<0.001$ \\
\hline Surgery & & & $<0.001$ \\
\hline None & $224(3.4)$ & $529(2.8)$ & \\
\hline Breast conservation & $4,030(60.6)$ & $12,386(64.8)$ & \\
\hline Mastectomy & $2,395(36)$ & $6,213(32.5)$ & \\
\hline Cardiovascular disease & I,93। (29) & $5,052(26.4)$ & $<0.001$ \\
\hline Diabetes & I,800 (27.1) & $5,647(29.5)$ & $<0.001$ \\
\hline
\end{tabular}

Note: Data presented as No. (\%) unless otherwise noted. Abbreviation: SD, standard deviation. 
Table 2 Association of Endocrine Therapy in the Treatment of Breast Cancer and Dementia Risk $(n=25,777)$

\begin{tabular}{|l|c|c|c|}
\hline Model & HR & $\mathbf{9 5 \%} \mathbf{~ C l}$ & P-value \\
\hline Univariable & 0.77 & $0.7 I-0.84$ & $<0.001$ \\
\hline Adjusted* & 0.91 & $0.83-0.98$ & 0.018 \\
\hline Adjusted with competing risk & 0.98 & $0.90-1.07$ & 0.631 \\
Al only ( $n=15,058)$ & 0.94 & $0.86-1.03$ & 1.00 \\
SERM only $(n=1,762)$ & 1.18 & $1.03-1.36$ & 0.02 \\
SERM + Al $(n=2,308)$ & 1.00 & $0.87-1.15$ & 0.98 \\
\hline
\end{tabular}

Notes: *Adjusted for age, race, region, marital status, T stage, Index, treatment with radiation, surgery, or chemotherapy. 'Competing risk of death.

Abbreviations: $\mathrm{Al}$, aromatase inhibitor; $\mathrm{Cl}$, confidence interval; $\mathrm{HR}$, hazards ratio; SERM, selective estrogen receptor modulator.

for dementia. Modeling the risk of the outcome similarly for individuals lost to follow-up and who are deceased may not be appropriate, particularly when examining older populations at greater risk of death from any cause. Competing risk analysis accounts for the probability of the outcome, here dementia, in the presence of a competing risk, here death, and avoids overestimation of association effects. ${ }^{7}$ Our ability to replicate a protective effect of ET on dementia risk, which then completely attenuates when implementing a competing risk model, suggests that prior studies may have overestimated the association of ET and dementia risk by not accounting for the competing risk of death.

Prior studies have shown conflicting results regarding the association of ET in the treatment of breast cancer with altered dementia risk. A population-based study in Taiwan examined 24,197 patients diagnosed with breast cancer and found that tamoxifen users had a $17 \%$ relative reduction in dementia risk. ${ }^{3}$ Interestingly, they found that a longer duration tamoxifen resulted in further increased risk and they found no association between dementia risk and treatment with AIs. Whether SERMs act on the brain to impact brain function, an effect which could vary based on menopausal status, remains an area of active investigation. ${ }^{11,12}$ SERMs are known to have tissue specific estrogenic and anti-estrogenic effects and therefore the potential to both positively and adversely impact cognitive function. Conversely, a Danish nationwide medical registries study examined 16,419 patients with breast cancer and found a weak association between tamoxifen therapy and dementia risk (HR, $1.4 ; 95 \% \mathrm{CI}, 1.0-1.9)$ that completely attenuated when accounting for death as a competing risk (SHR, 1.0; 95\% CI 0.76-1.4). ${ }^{4} \mathrm{~A}$ recent study using Humana claims data examined 326,485 women with breast cancer and found a $12 \%$ relative dementia risk reduction in adjusted models. However, this study was unable to account for the competing risk of death. ${ }^{5}$

Our study has limitations. Our findings may not be generalizable to younger populations as we only examined individuals $\geq 66$ years of age. Additionally, our ability to examine the association of SERM and dementia risk was limited by low power and should be further investigated in future studies. Our study design was retrospective in nature and therefore may still be subject to bias and residual confounding. We were unable to account for smoking in our adjusted models, which is a potential confounding factor. Additionally, our study had a relatively short duration of follow-up and it is possible that we were therefore unable to detect longer term impacts of ET on dementia risk.

In conclusion, when accounting for common sources of bias and confounding, in particular competing risks, we did not find evidence to support an association between ET in the definitive treatment of non-metastatic breast cancer and dementia risk. Future studies powered to explore SERM use and dementia risk, particularly among younger individuals, are needed.

\section{Acknowledgments}

The research was supported, in part, by National Institutes of Health CCSG P30 CA016672. Dr. Nead and Dr. Wehner are Cancer Prevention Research Institute of Texas (CPRIT) Scholars in Cancer Research. Dr. Nead is supported by CPRIT RR190077. Dr. Wehner is supported by CPRIT FP9178. Dr. Giordano is supported by CPRIT RP160674 and Komen SAC150061. The funding organizations had no role in the design and conduct of the study; collection, management, analysis, and interpretation of the data; preparation, review, or approval of the manuscript; and decision to submit the manuscript for publication. Dr. Nead had full access to all the data in the study and takes responsibility for the integrity of the data and the accuracy of the data analysis. Dr. Niu conducted and is responsible for the data analysis.

\section{Disclosure}

The authors report no conflicts of interest in this work.

\section{References}

1. Siegel RL, Miller KD, Jemal A. Cancer statistics, 2020. CA Cancer J Clin. 2020;70(1):7-30. doi:10.3322/caac.21590

2. Tremont A, Lu J, Cole JT. Endocrine therapy for early breast cancer: updated review. Ochsner J. 2017;17(4):405-411. 
3. Sun LM, Chen HJ, Liang JA, Kao CH. Long-term use of tamoxifen reduces the risk of dementia: a nationwide population-based cohort study. QJM. 2016;109(2):103-109. doi:10.1093/qjmed/ hev072

4. Ording AG, Jensen AB, Cronin-Fenton D, Pedersen L, Sorensen HT, Lash TL. Null association between tamoxifen use and dementia in Danish breast cancer patients. Cancer Epidemiol Biomarkers Prev. 2013;22(5):993-996. doi:10.1158/1055-9965.EPI-13-0139

5. Branigan GL, Soto M, Neumayer L, Rodgers K, Brinton RD. Association between hormone-modulating breast cancer therapies and incidence of neurodegenerative outcomes for women with breast cancer. JAMA Netw Open. 2020;3(3):e201541. doi:10.1001/ jamanetworkopen.2020.1541

6. Levesque LE, Hanley JA, Kezouh A, Suissa S. Problem of immortal time bias in cohort studies: example using statins for preventing progression of diabetes. BMJ. 2010;340:b5087. doi:10.1136/bmj.b5087

7. Berry SD, Ngo L, Samelson EJ, Kiel DP. Competing risk of death: an important consideration in studies of older adults. J Am Geriatr Soc. 2010;58(4):783-787. doi:10.1111/j.1532-5415.2010.02767.x
8. Williams SB, Huo J, Chamie K, et al. Discerning the survival advantage among patients with prostate cancer who undergo radical prostatectomy or radiotherapy: the limitations of cancer registry data. Cancer. 2017;123(9):1617-1624. doi:10.1002/cncr.30506

9. Fine JP, Gray RJ. A proportional hazards model for the subdistribution of a competing risk. J Am Stat Assoc. 1997;94:496-509. doi:10. 1080/01621459.1999.10474144

10. Matthews KA, Xu W, Gaglioti AH, et al. Racial and ethnic estimates of Alzheimer's disease and related dementias in the United States (2015-2060) in adults aged $\geq 65$ years. Alzheimers Dement. 2019;15 (1):17-24. doi:10.1016/j.jalz.2018.06.3063

11. Eberling JL, Wu C, Tong-Turnbeaugh R, Jagust WJ. Estrogen- and tamoxifen-associated effects on brain structure and function. Neuroimage. 2004;21(1):364-371. doi:10.1016/j.neuroimage.2003. 08.037

12. Silva I, Mello LE, Freymuller E, Haidar MA, Baracat EC. Estrogen, progestogen and tamoxifen increase synaptic density of the hippocampus of ovariectomized rats. Neurosci Lett. 2000;291(3):183-186. doi:10.1016/S0304-3940(00)01410-5

\section{Publish your work in this journal}

Breast Cancer - Targets and Therapy is an international, peer-reviewed open access journal focusing on breast cancer research, identification of therapeutic targets and the optimal use of preventative and integrated treatment interventions to achieve improved outcomes, enhanced survival and quality of life for the cancer patient.
The manuscript management system is completely online and includes a very quick and fair peer-review system, which is all easy to use. Visit http://www.dovepress.com/testimonials.php to read real quotes from published authors. 